\title{
Effect of Foot Orthoses on Children With Lower Extremity Growing Pains
}

\author{
Hong-Jae Lee, $\mathrm{MD}^{1}$, Kil-Byung Lim, $\mathrm{MD}^{1}$, JeeHyun Yoo, $\mathrm{MD}^{1}$, \\ Sung-Won Yoon, $\mathrm{MD}^{1}$, Tae-Ho Jeong, $\mathrm{MS}^{2}$ \\ ${ }^{1}$ Department of Physical Medicine and Rehabilitation, \\ ${ }^{2}$ Institute of Sports Rehabilitation, Inje University Ilsan Paik Hospital, Goyang, Korea
}

Objective To evaluate the effect of custom molded foot orthoses on children with growing pains of the lower extremities.

Methods Children with growing pains were recruited during a clinic visit. None had any bony deformities, inflammatory joint disease or depression. Resting calcaneal stance position (RCSP) and calcaneal pitch angle were measured, and individual custom molded rigid foot orthoses were prescribed using the inverted orthotic technique to control for foot overpronation using the RCSP angle. Pain sites, degree and frequency of pain, and balance ability were evaluated using Balance Master computerized posturography prior to foot orthosis fitting, and 1 and 3 months later.

Results Twenty children completed the study. Seventeen (75\%) had overpronated feet. Significant improvements were noted after 1 and 3 months in pain degree and frequency, and after 3 months in balancing ability.

Conclusion The use of custom molded foot orthoses is a good method for treating children with multiple musculoskeletal pains in the leg.

Keywords Musculoskeletal pain, Pediatrics, Orthotic devices, Postural balance

\section{INTRODUCTION}

Musculoskeletal pains are common in pediatric patients and range from benign conditions to cases requiring immediate attention [1]. Among them, growing pains,

Received June 17, 2014; Accepted October 2, 2014

Corresponding author: Sung-Won Yoon

Department of Physical Medicine and Rehabilitation, Sports Medicine Center, Inje University Ilsan Paik Hospital, 170 Juhwa-ro, Ilsanseo-gu, Goyang 411-706, Korea

Tel: +82-31-910-7440, Fax: +82-31-910-7446, E-mail: 19534@paik.ac.kr

(c) This is an open-access article distributed under the terms of the Creative Commons Attribution Non-Commercial License (http://creativecommons. org/licenses/by-nc/3.0) which permits unrestricted noncommercial use, distribution, and reproduction in any medium, provided the original work is properly cited.

Copyright $\odot 2015$ by Korean Academy of Rehabilitation Medicine first recognized in 1823, are unexplainable, recursive, and self-limiting musculoskeletal pains [2]. The pains usually occur at night and are usually accompanied by no clinical signs of inflammation. Growing pains also improve at the end of childhood $[3,4]$.

The prevalence rate of growing pains is reportedly $2.6 \%-49.4 \%$ [5-11], and known causes include growth [9], hypermobility and fatigue [12-15], anatomical/biomechanical factors $[16,17]$, and psychological problems $[9,18]$. While clinicians face these symptoms relatively more often, the cause of the disease is not clearly understood and appropriate treatments are not always provided. There is no special test for diagnosing growing pains. Instead, in most cases, clinical decisions are made ac- 
cording to inclusive or exclusive criteria [15,19-21]. Criteria for the diagnosis of growing pains were suggested in a cohort study of 720 students divided into three groups [9]. The criteria included pain lasting longer than 3 months, symptom-free periods of several days to several months with only intermittent pain episodes, pain occurring in the late afternoon or during sleep, lack of arthralgia, pain so severe that it interferes with sleep, and normal X-ray, blood test, physical examination findings. Other suggested criteria to diagnose growing pains included pains that are intermittent and bilateral, localized at the front thigh or in the shin mostly in the afternoon, and no abnormal findings on radiological or hematological tests [16].

Massage, nonsteroidal anti-inflammatory drugs, and orthoses are helpful for patients who complain of benign limb pain, for whom initial reassurance with a comment that symptoms should improve with age helps [1]. However, appropriate treatments to remove causes have not yet been established, and studies of treatment are lacking, even though various methods like those described above have been proposed to control growing pains.

Anatomical factors, fatigue, and psychological factors are thought to be causes of growing pains. Hypermobility and flat feet have been proposed anatomical factors of growing pains [16]. Presently, we studied children who complained of recursive growing pains in the lower extremities, investigated malalignment conditions of the lower extremities, and comparatively analyzed the frequency of pain occurrence, pain degree, and changes in their ability to perform static and dynamic balancing and functional activities after wearing customized foot orthoses that controlled biomechanical malalignment of the feet and lower limbs.

\section{MATERIALS AND METHODS}

\section{Study subjects}

Pediatric patients clinically diagnosed with growing pains who visited our hospital's foot clinic complaining of musculoskeletal pains in the lower extremities between June 2012 and December 2013 were recruited. Since there are no established criteria for diagnosis of growing pains, patients were selected with more rigorous clinical criteria than those previously proposed [9]. Accordingly, inclusion criteria were complaint of pains in the lower limbs mostly in the afternoon or at night, pain-related awakening at night, complaint of pains in the lower limbs four times a month or an average of at least once a week for the past 6 months, capability of normal daily activities like running during pain-free periods, and school age $(>6$ years) and ability to perform balancing tests. Exclusion criteria were bone fractures or deformities detected on $\mathrm{X}$-ray images, neurologic anomalies, infectious diseases, hemostatic disorders, malignant diseases, and current treatment for depression.

\section{Methods}

Biomechanical and radiological tests and the use of foot orthoses

Calcaneal pitch angle and resting calcaneal stance position were measured by foot X-rays and biomechanical tests, respectively. To measure the resting calcaneal stance position, the bimanual technique was used. The midpoints of the calcaneus were connected in the coronal plane with the subjects lying prone. After partitioning the calcaneus in this way, the angle between the perpendicular line to the ground and the bisector of the calcaneus was measured with subjects standing to fit the ambulation angle and ambulation base. Positive and negative values in the resting calcaneal stance position indicated supination and pronation, respectively. When a biomechanical abnormality like overpronation was found, a customized foot orthosis was prescribed using the inverted orthotic technique [22] (Fig. 1). For example, when the initial RCSP were $-3^{\circ}$ to $-4^{\circ},-5^{\circ}$ to $-6^{\circ}$, and $-7^{\circ}$ to $-8^{\circ}$, the inverted orthotic technique prescribed $15^{\circ}, 20^{\circ}$, and $25^{\circ}$ angles, respectively. For a RCSP of $-9^{\circ}$ to $-10^{\circ}$ but lower than $-11^{\circ}$ (severe type), $30^{\circ}$ and $35^{\circ}$ inverted orthotic technique with lateral long flanges were prescribed, respectively.

Evaluation of pain frequency, sites, and degrees in the feet and lower extremities

Sites of pains in the lower extremities, as well as frequency and degrees of pains were compared with before treatment, 1 month post-treatment, and 3 months posttreatment. The visual analog scale (VAS) was used to evaluate pain frequency.

\section{Evaluation of balancing ability}

A Balance Master system (NeuroCom, Clackamas, OR, USA) was used to evaluate balancing ability including 

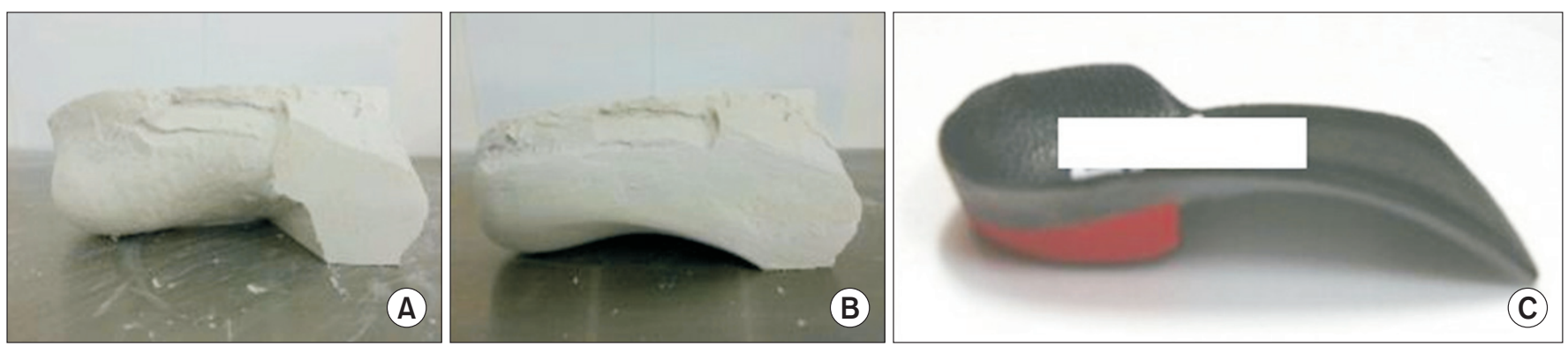

Fig. 1. Fabrication of custom molded rigid foot orthoses made using the inverted orthotic technique. (A) Inverting of the positive cast and platforming of the forefoot. (B) Remodeling of the plantar arch from the sustentaculum tali to the first metatarsal head. (C) The finalized orthosis.

static, dynamic, and functional test methods. In detail, sensory limitation assessment items included static balancing ability, motor limitation assessment items for dynamic balancing ability, and functional limitation assessment items for functional balancing ability. These tests were performed before and 3 months after treatment with orthoses to compare short-term effects. The reason why the tests were performed after wearing foot orthoses after 3 months was because the children performed daily activities while continuously wearing foot orthoses, so the balancing test was intended to be conducted in the real-life setting of pediatric patients wearing foot orthoses.

Sensory limitation assessment for static balancing ability As subsections of sensory limitation assessment of this study, modified clinical test sensory interaction on balance (mCTSIB) and the unilateral stance were measured. For the MCTSIB, foam-interfering proprioception was placed on a force plate and subjects stood on it and maintained their postures with eyes opened or closed for 10 seconds each for measurement of the center of gravity (COG) sway velocity $(\% / s)$. For the unilateral stance test, subjects stood on one leg and tried to maintain their posture with their eyes opened or closed for 10 seconds on a force plate to measure the COG sway velocity. Each test was repeated three times and the mean values were calculated.

\section{Motor limitation assessment for dynamic balancing ability}

For motor limitation assessment, five subsections of stability limits were tested. The limits of stability test examined how far subjects intentionally moved their COG to the left or right from the original center, for which reac- tion time, movement velocity moving the COG, endpoint and maximum excursion, and directional control were measured. Tests were repeated three times and the mean values were calculated.

\section{Functional limitation assessment of functional balanc-} ing ability

As subsections of this test, the step/quick turn and tandem gait were measured. For the step/quick turn, subjects waited at the starting point, stepped onto a force plate with one leg at the examiner's starting signal, turned around $180^{\circ}$ after two steps, and returned to the starting point. During this process, turn sway $\left({ }^{\circ}\right)$ of the COG and turn time (s) were measured. For the tandem walk test, subjects walked on a test plate with the toe and the foot heel touching the plate for examination of the walking characteristics. The measured values were step width, step speed, and end sway.

\section{Analyses}

SPSS ver. 21.0 (IBM SPSS, Armonk, NY, USA) was used for statistical analyses, repeated-measures analysis of variance was used to compare pain degree and frequency (three time points comparison) at each time point before and after wearing foot orthoses, while the Wilcoxon signed-rank test was used to analyze the degree of balancing ability change (two time points comparison).

\section{RESULTS}

\section{Subject general characteristics}

Fifty-six children satisfied the clinical selection criteria. Twenty-one subjects were $>6$ years. Twenty of these pediatric patients (seven boys, 13 girls) completed the 


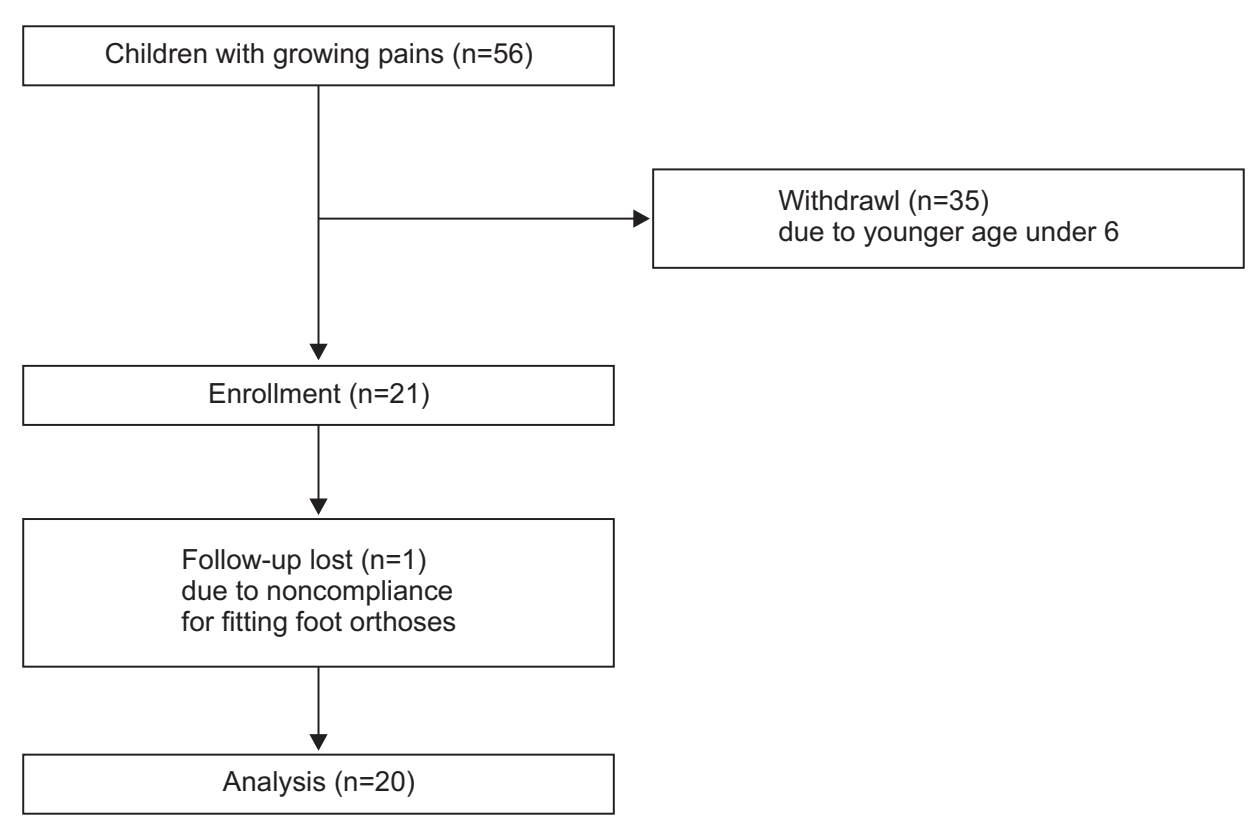

Fig. 2. Flow chart of the patients.

Table 1. General characteristics of the subjects $(n=20)$

\begin{tabular}{lc}
\hline \multicolumn{1}{c}{ Characteristic } & Value \\
\hline Sex (male:female) & $7: 13$ \\
\hline Age $(\mathrm{yr})$ & $9.10 \pm 2.32$ \\
\hline Height $(\mathrm{cm})$ & $134.35 \pm 14.05$ \\
\hline Weight $(\mathrm{kg})$ & $30.95 \pm 9.34$ \\
\hline
\end{tabular}

Values are presented as mean \pm standard deviation or number.

3-month study (Fig. 2). The average age, height, and weight of children completed the study was $9.10 \pm 2.32$ years, $134.35 \pm 14.05 \mathrm{~cm}$, and $30.95 \pm 9.34 \mathrm{~kg}$, respectively (Table 1).

\section{Biomechanical and radiological test findings}

In the biomechanical test, 17 of the 20 (85\%) children showed at least one foot with overpronation (RCSP $\left.\leq-5^{\circ}\right)$. The rest had a lower RCSP $\left(-3^{\circ},-4^{\circ}\right)$, and no child had a normal RCSP (RCSP $\left.\geq-2^{\circ}\right)$. The average RCSP of all participants was $-6.4^{\circ}$ on the left and $-8.1^{\circ}$ on the right. On the X-ray test, 15 of the $20(75 \%)$ children exhibited findings (calcaneal pitch angle $<20^{\circ}$ ) indicative of flat feet, and 11 children had a pitch angle $<15^{\circ}$. The average calcaneal pitch angle of the entire participant group was $16.40^{\circ}$ on the left and $17.11^{\circ}$ on the right (Table 2).
Table 2. RCSP and calcaneal pitch angle

\begin{tabular}{lcc}
\hline & Right & Left \\
\hline RCSP $\left({ }^{\circ}\right)$ & $-8.10 \pm 4.21$ & $-6.40 \pm 3.34$ \\
Calcaneal pitch angle $\left(^{\circ}\right)$ & $16.40 \pm 5.30$ & $17.11 \pm 5.30$
\end{tabular}

Values are presented as mean \pm standard deviation. RCSP, resting calcaneal stance position.

Table 3. Location of pain

\begin{tabular}{lc}
\hline \multicolumn{1}{|c}{ Location } & No. of patients \\
\hline Calf & 10 \\
\hline Sole & 7 \\
\hline Anterior knee & 6 \\
\hline Anterior ankle & 5 \\
\hline Anterior tibia & 3 \\
\hline Heel & 3 \\
\hline Posterior knee & 2 \\
\hline Etc. & 2 \\
\hline
\end{tabular}

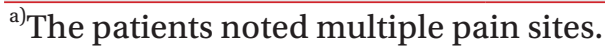

\section{Changes in pain regions, degrees, and frequencies}

Pain sites were identified by the subjects as the calf (10 children), sole (seven children), anterior knee around the patella (six children), and anterior ankle (five children). Some pediatric patients complained of pain in the anterior tibia, posterior knee, and posterior heel (Table 3). Fourteen of the $20(70 \%)$ children complained of pain in more than two regions. 
Compared with before treatment $(12.45 \pm 10.57$ per month), pain frequency was decreased significantly at 1 month after treatment $(8.90 \pm 10.95$ per month) and 3 months after treatment (5.40 \pm 8.51 per month) (Fig. 3). Since the frequency of pain occurrence was statistically significant $(\mathrm{p}=0.001)$ over time, pain frequency can be concluded to have occurred in a temporally linear pattern. Statistically significant differences were evident in comparisons before treatment and 1 month after treatment ( $p=0.006)$, and before treatment and 3 months after treatment ( $\mathrm{p}=0.003)$. However, the comparison between 1 month and 3 months after treatment was statistically insignificant $(p=0.164)$. These findings indicate that frequency of pain occurrence was improved within 1 month after wearing foot orthoses.

Degree of pain in the most complained sites evaluated using VAS displayed a statistically significant improvement compared with before treatment $(6.25 \pm 2.00), 1$ month after treatment $(3.60 \pm 2.50)$, and 3 months after

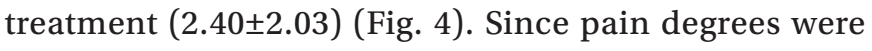
statistically significant $(\mathrm{p}<0.001)$ over time, they can be concluded to have followed a temporally linear pattern. Significant differences were evident in comparisons between before treatment and 1 month after treatment $(\mathrm{p}<$ $0.001)$, before treatment and 3 months after treatment $(\mathrm{p}<0.001)$, and 1 and 3 months after treatment $(\mathrm{p}=0.023)$. These findings mean that pain degrees in the most affected sites significantly decreased after both 1 and 3 months.

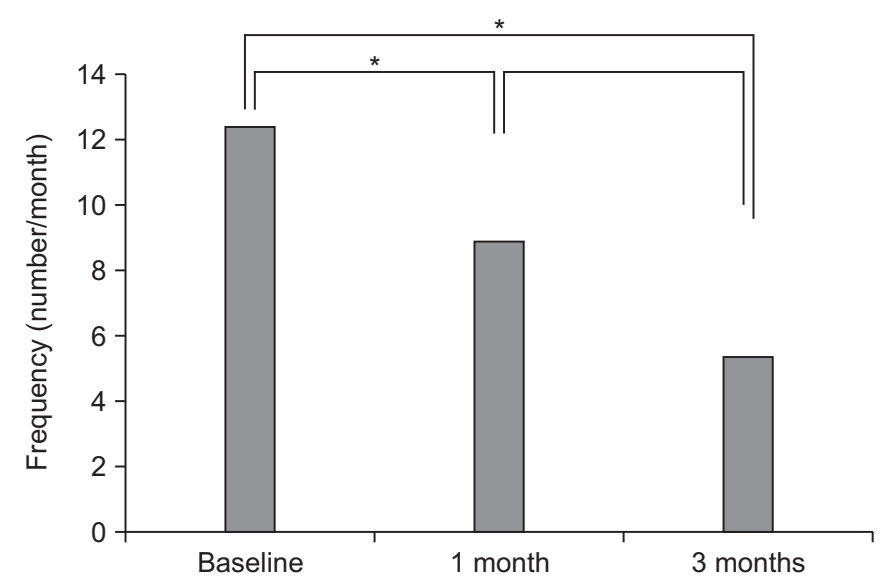

Fig. 3. Frequency of pain occurrence. The p-values were derived using repeated-measures analysis of variance. ${ }^{*} \mathrm{p}<0.01$.

\section{Change in balancing ability \\ Static balance}

In a unilateral stance test with eyes opened, when the COG sway velocity was compared before versus 3 months after treatment, it decreased statistically significantly from $1.50 \pm 0.56(\%)$ s $) 1.22 \pm 0.62(\% / \mathrm{s})$ during standing on the left foot. Comparing before and after treatment with eyes closed, the velocity of the left decreased from $3.45 \pm 1.7(\mathrm{o} / \mathrm{s})$ to $2.63 \pm 1.06(\% / \mathrm{s})$, while that of the right decreased from $3.52 \pm 1.45(\% / \mathrm{s})$ to $2.61 \pm 1.03(\%)$, so that both cases showed statistically significant improvements (Table 4).

\section{Dynamic balance}

The five limits of stability subsections were measured with the child moving in four directions (forward, backward, left, and right). In the movement velocity domain, forward movement speeds improved significantly from $5.08 \pm 1.43(\% / \mathrm{s})$ to $6.48 \pm 1.52(\% / \mathrm{s})$, while horizontal movement also showed a statistically significant improvement. In addition, the endpoint excursion area performed to the left showed a statistically significant improvement from $93.25 \% \pm 23.61 \%$ to $105.55 \% \pm 21.94 \%$. The maximum excursion domain also resulted in statistically significant improvement from $110.75 \% \pm 18.47 \%$ to $120.15 \% \pm 16.05 \%$ (Table 4).

\section{Functional balance}

Although no significant change was seen on the step/ quick turn test, the tandem walk test showed a statisti-

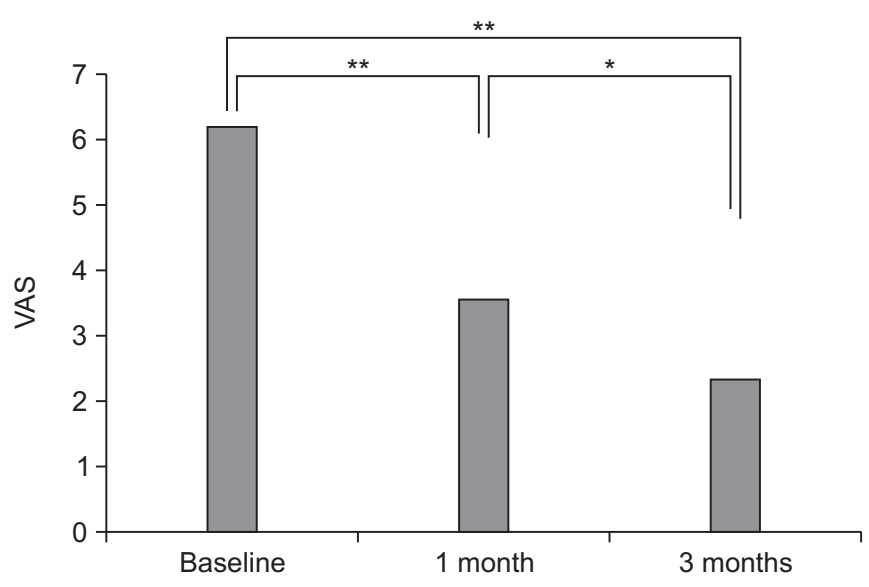

Fig. 4. Visual analog scale (VAS) of pain in the main pain site. The $p$-values were derived using repeated-measures analysis of variance. ${ }^{*} \mathrm{p}<0.05,{ }^{* *} \mathrm{p}<0.001$. 
Hong-Jae Lee, et al.

Table 4. Comparison of static, dynamic, and functional balancing ability after fitting the foot orthoses

\begin{tabular}{|c|c|c|c|}
\hline Parameter & Before & 3 months & p-value \\
\hline \multicolumn{4}{|l|}{ Static balance } \\
\hline \multicolumn{4}{|c|}{ Unilateral stance - COG sway velocity $(\mathrm{o} / \mathrm{s})$} \\
\hline Left EO & $1.50 \pm 0.56$ & $1.22 \pm 0.62$ & $0.012^{*}$ \\
\hline Right EO & $1.51 \pm 0.66$ & $1.44 \pm 0.90$ & 0.860 \\
\hline Left EC & $3.45 \pm 1.72$ & $2.63 \pm 1.06$ & $0.022^{*}$ \\
\hline Right EC & $3.52 \pm 1.45$ & $2.61 \pm 1.03$ & $0.011^{*}$ \\
\hline \multicolumn{4}{|l|}{ Dynamic balance } \\
\hline \multicolumn{4}{|l|}{ Movement velocity (o/s) } \\
\hline Forward & $5.08 \pm 1.43$ & $6.48 \pm 1.52$ & $0.033^{*}$ \\
\hline Back & $4.63 \pm 1.71$ & $4.09 \pm 1.20$ & 0.240 \\
\hline Right & $6.86 \pm 2.31$ & $8.55 \pm 3.21$ & $0.044^{*}$ \\
\hline Left & $6.72 \pm 2.11$ & $8.72 \pm 3.81$ & $0.046^{*}$ \\
\hline \multicolumn{4}{|l|}{ Endpoint (\%) } \\
\hline Forward & $69.15 \pm 19.70$ & $78.75 \pm 11.32$ & 0.180 \\
\hline Back & $64.05 \pm 19.38$ & $68.60 \pm 14.95$ & 0.350 \\
\hline Right & $85.85 \pm 28.43$ & $102.65 \pm 27.00$ & 0.090 \\
\hline Left & $93.25 \pm 23.61$ & $105.55 \pm 21.94$ & $0.048^{*}$ \\
\hline \multicolumn{4}{|l|}{ Max excursion (\%) } \\
\hline Forward & $94.35 \pm 18.60$ & $99.75 \pm 12.32$ & 0.410 \\
\hline Back & $82.10 \pm 21.47$ & $92.75 \pm 15.01$ & 0.050 \\
\hline Right & $110.60 \pm 20.31$ & $122.80 \pm 19.42$ & 0.060 \\
\hline Left & $110.75 \pm 18.47$ & $120.15 \pm 16.05$ & $0.030^{*}$ \\
\hline \multicolumn{4}{|l|}{ Functional balance } \\
\hline \multicolumn{4}{|l|}{ Tandem walk } \\
\hline Step width $(\mathrm{cm})$ & $8.37 \pm 2.10$ & $9.09 \pm 3.07$ & 0.410 \\
\hline Speed $(\mathrm{cm} / \mathrm{s})$ & $33.69 \pm 8.83$ & $39.27 \pm 9.78$ & $0.022^{*}$ \\
\hline End sway (o/s) & $6.59 \pm 1.82$ & $6.85 \pm 1.91$ & 0.990 \\
\hline
\end{tabular}

Values are presented as mean \pm standard deviation.

COG, center of gravity; EO, eye open; EC, eye closed.

${ }^{*} \mathrm{p}<0.05$ by Wilcoxon signed-rank test.

cally significant improvement from $33.69 \pm 8.83(\mathrm{~cm} / \mathrm{s})$ to $39.27 \pm 9.78(\mathrm{~cm} / \mathrm{s})$ in the step speed domain (Table 4$)$.

\section{DISCUSSION}

Pediatric patients who complained of growing pains wore foot orthoses for 3 months. When the frequency of growing pains and degree of the pains were evaluated after 3 months, both exhibited statistically significant improvements. Growing pains are frequently found in the age groups of childhood and adolescence, and are often faced clinically.
Massage, stretching, or drug administration are often used to lessen symptoms [13]. A small case study attempting to control growing pains through a biomechanical approach to correct overpronated feet with foot orthoses and reported that foot orthoses effectively controlled growing pains [16]. A later study argued that anatomical factors like foot posture are irrelevant to growing pains [23].

This present hypothesis was that if customized foot orthoses using the inverted orthotic technique were used in pediatric patients complaining of growing pains to correct foot misalignment and instability, growing pains in 
the feet and lower extremities would be reduced. Seventeen of $20(75 \%)$ subjects had overpronated feet with an RCSP $\leq-5^{\circ}$, and exhibited statistically significant improvements in pain frequency and degree 1 and 3 months after wearing foot orthoses (Figs. 3, 4). In addition, since all pediatric patients complained of chronic and recursive pains for more than 6 months, it was difficult to expect natural improvements of growing pains within 1 month. So, reduction of growing pains is considered to be an effect of foot orthoses.

Growing pains caused by fatigue or myalgia in the muscles of the lower limbs, rather than by damage to the bone itself [12-15]. Myalgia is caused by overuse of the muscle in the lower limbs by excessive training, severe fatigue in proximal parts of tibialis posterior muscle or tibialis anterior muscle by excessive pronation, and hyperextension caused by a lack of flexibility in the peroneal muscle [24].

Since there was no athlete among the participating children, the growing pains were not likely correlated with excessive training. Non-athlete children are rarely exposed to the situation of excessive training. Therefore, it is assumed that growing pains are likely correlated with fatigue or pain in the peroneal muscle that occurred in tibialis posterior or tibialis anterior muscles by excessive pronation. A number of children were excluded due to age. Most of the pediatric patients who were selected for this study exhibited overpronation $\left(\operatorname{RCSP} \leq-5^{\circ}\right)$ on biomechanical testing. Since the peroneal muscle is the most usual site of pains, it seems that imposing extension stress on the tibialis posterior muscle was effective. Since the tibialis posterior muscle, which is located at the center of the peroneal portion of the leg, functions as the primary muscle that maintains the arch of the foot and controls overpronation, muscle fatigue, and myalgia are highly prone to occur within this muscle. In addition, since overpronated feet of children are mostly flexible flat feet, mechanical instability of the feet occurs frequently. In this foot condition, intrinsic and extrinsic muscles of the foot need to be more functional, so that the risk of myalgia would be increased due to overuse of the corresponding muscles.

When customized foot orthoses produced using the inverted orthotic technique are applied to overpronated feet of pediatric patients, they control overpronation and limit the overpronation response and secondary response in the lower extremities, leading to reduced fatigue or pain in the peroneal muscle including the tibialis posterior and anterior muscles. Customized foot orthoses are also thought to decrease the secondary genu valgum of the knee joint and maltracking of the knee joint caused by overpronation, resulting in eliminated femoral patellar pain (the most common cause of front gonalgia). In other words, customized foot orthoses produced using the inverted orthotic technique are thought to confer biomechanical stability to the feet and the lower extremities, resulting in a significant reduction of pain frequency and degree in the lower limbs.

In the evaluation of static balancing ability, the COG sway velocity on a unilateral stance test was significantly reduced. In the evaluation of dynamic balancing ability, movement velocity area and endpoint and max excursion area showed statistically significant improvements. In the evaluation of functional balancing ability, step speed on the tandem walk test exhibited statistically significant improvements (Table 4).

This study was performed to determine whether posture maintenance and balancing are improved by the foot orthoses produced using the inverted orthotic technique to provide the feet and lower extremities biomechanical stability. In the evaluation of static balancing ability (posture maintaining ability), sway was consistently reduced in multiple directions on the COG sway velocity test that was performed with each participant standing on one leg. In particular, sway was significantly reduced when the compensation for visual sense was removed by closing the eyes. Maintaining the posture of standing with one leg with eyes closed is a situation that requires an individual to maintain their posture only with proprioception of the foot and the ankle and tactile sense of the sole. Improvement of balancing ability after wearing foot orthoses in that situation indicates that wearing foot orthoses further stimulated the proprioceptive and tactile senses of the sole, or at least provided better information to them. In fact, foot orthoses produced using the inverted orthotic technique directed the subtalar joint to a near-neutral position and the midtarsal joint close to the normal position. By restoring joint position this way, high-quality afferent position sense information should be transduced from the ankle joints and tarsal bone to the central nervous system.

Another reason the posture maintaining ability was im- 
proved was because the foot orthoses in this study conferred biomechanical stability of the feet. Children with flexible flat feet frequently experience biomechanical instability within the bones of the feet. Thus, a customized foot orthoses made of rigid plastic should lift up the entire arch of the ankle and foot including subtalar and midtarsal joints, resulting in great reduction of mechanodynamic instability of the bones of the feet. A reduction of mechanodynamic instability will be able to decrease fatigue in the intrinsic and extrinsic muscles that control foot position.

Use of foot orthoses can improve joint position sense by increasing the afferent feedback from the skin receptors within the foot and ankle [25]. Since the inverted orthotic technique was designed to place major joints in the foot into normal positions and confer mechanical stability to the bones of foot and the joints, the use of the inverted orthotic technique using a solid material helped improve balancing ability through transduction of normal afferent signals from proprioception receptors of the ankle or skin receptors of the sole.

Among the analyses used to test dynamic balancing ability, movement velocity, endpoint excursion distance, and maximum excursion distance also displayed significant improvements. In these evaluations, subjects actively moved their COG following the order provided on a computer screen. The improvements in these sections meant that the body moved as the subject intended, which was likely enabled by mechanical stability of the feet, and appropriate transmission of the afferent joint position sense signal of the foot and ankle.

In the tandem walk test for evaluating functional balancing ability, step speed improved significantly. A significant improvement in test results meant that the foot with the balancing function of touching the ground was better able to support the body when the orthoses was applied. This was believed to be enabled by mechanical stability and proper afferent signal transduction from the foot and the ankle. The customized foot orthoses produced by the inverted orthotic technique markedly reduced pain frequency and degree in pediatric patients with growing pains and improved their static, dynamic, and functional balancing abilities.

This study had a few limitations. First, it had a small cohort size (20 subjects). Second, it was necessary to limit participant age to evaluate balance ability. If only pain change data had been used without an age limitation, a larger group (50 children) would have contributed to the results. In addition, the period of follow-up was 3 months, which is relatively short. As a follow-up study, results obtained 1 year later will be published. In this study, evaluation of balancing ability 3 months after treatment was performed with subjects wearing foot orthoses. As a compensatory study, balancing ability will be evaluated with and without wearing it in the followup study to find out the effect of wearing the foot orthosis for 1 year. In addition, this study did not consider patient body type or body mass index. However, since none of our patients appeared obese, we believe that this limitation did not affect the results. Future follow-up studies should be performed to compensate for these limitations.

In this study, $75 \%$ of pediatric patients complaining of growing pains had overpronated feet. When they wore customized foot orthoses produced using the inverted orthotic technique, pain frequency and degree were significantly improved. Static, dynamic, and functional balancing abilities also improved. Therefore, custom molded foot orthoses using the inverted orthotic technique will be an excellent treatment method for pediatric patients complaining of growing pains.

\section{CONFLICT OF INTEREST}

No potential conflict of interest relevant to this article was reported.

\section{REFERENCES}

1. Weiser P. Approach to the patient with noninflammatory musculoskeletal pain. Pediatr Clin North Am 2012;59:471-92.

2. Duchamp M. Maladies de la croissance. Paris: (Fain); 1823.

3. Uziel Y, Hashkes PJ. Growing pains in children. Harefuah 2008;147:809-11, 836.

4. Lowe RM, Hashkes PJ. Growing pains: a noninflammatory pain syndrome of early childhood. Nat Clin Pract Rheumatol 2008;4:542-9.

5. Oster J, Nielsen A. Growing pains. A clinical investigation of a school population. Acta Paediatr Scand 1972; 61:329-34.

6. Williams MF. Rheumatic conditions in school-chil- 
dren. Lancet 1928;211:720-1.

7. Mikkelsson M, Salminen JJ, Kautiainen H. Non-specific musculoskeletal pain in preadolescents: prevalence and 1-year persistence. Pain 1997;73:29-35.

8. Brenning R. Growing pain. Acta Soc Med Ups 1960; 65:185-201.

9. Naish JM, Apley J. "Growing pains": a clinical study of non-arthritic limb pains in children. Arch Dis Child 1951;26:134-40.

10. Abu-Arafeh I, Russell G. Recurrent limb pain in schoolchildren. Arch Dis Child 1996;74:336-9.

11. Oberklaid F, Amos D, Liu C, Jarman F, Sanson A, Prior M. "Growing pains": clinical and behavioral correlates in a community sample. J Dev Behav Pediatr 1997; 18:102-6.

12. Brenning R. “Growing pains". Sven Lakartidn 1960; 57:85-103.

13. Baxter MP, Dulberg C. "Growing pains" in childhood: a proposal for treatment. J Pediatr Orthop 1988;8:402-6.

14. Friedland O, Hashkes PJ, Jaber L, Cohen HA, Eliakim A, Wolach B, et al. Decreased bone speed of sound in children with growing pains measured by quantitative ultrasound. J Rheumatol 2005;32:1354-7.

15. Evans AM, Scutter SD, Lang LM, Dansie BR. "Growing pains" in young children: a study of the profile, experiences and quality of life issues of four to six year old children with recurrent leg pain. Foot (Edinb)
2006;16:120-4.

16. Evans AM. Relationship between "growing pains" and foot posture in children: single-case experimental designs in clinical practice. J Am Podiatr Med Assoc 2003; 93:111-7.

17. Hawksley JC. Growing pains in relation to rheumatism. Br Med J 1939;1:155-7.

18. Evans AM. Growing pains: contemporary knowledge and recommended practice. J Foot Ankle Res 2008;1:4.

19. Al-Khattat A, Campbell J. Recurrent limb pain in childhood ('growing pains'). Foot (Edinb) 2000;10: 117-23.

20. Peterson HA. Leg aches. Pediatr Clin North Am 1977; 24:731-6.

21. Peterson H. Growing pains. Pediatr Clin North Am 1986;33:1365-72.

22. Blake RL. Inverted functional orthosis. J Am Podiatr Med Assoc 1986;76:275-6.

23. Evans AM, Scutter SD. Are foot posture and functional health different in children with growing pains? Pediatr Int 2007;49:991-6.

24. Brukner P, Khan K. Brukner \& Khan's clinical sports medicine. 4th ed. New York: McGraw Hill; 2012.

25. Feuerbach JW, Grabiner MD, Koh TJ, Weiker GG. Effect of an ankle orthosis and ankle ligament anesthesia on ankle joint proprioception. Am J Sports Med 1994;22:223-9. 